\title{
CHARACTERIZATION OF A RECOVERABLE FLIGHT CONTROL COMPUTER SYSTEM
}

\author{
Mahyar Malekpour, NASA Langley Research Center, MS 130, Hampton, VA 23681 \\ Wilfredo Torres, NASA Langley Research Center, MS 130, Hampton, VA 23681 \\ Email: m.r.malekpour@larc.nasa.gov \\ w.torres-pomales@larc.nasa.gov
}

\begin{abstract}
The design and development of a Closed-Loop System to study and evaluate the performance of the Honeywell Recoverable Computer System (RCS) in electromagnetic environments (EME) is presented. The development of a Windows-based software package to handle the time-critical communication of data and commands between the RCS and flight simulation code in real-time, while meeting the stringent hard deadlines is also submitted. The performance results of the RCS and characteristics of its upset recovery scheme while exercising flight control laws under ideal conditions as well as in the presence of electromagnetic fields are also discussed.
\end{abstract}

\section{Introduction}

The problem of verifying the integrity of control computers in adverse as well as nominal operating environments is a key issue in the development, validation, certification, and operation of critical control systems for advanced aircraft. An adverse operating environment of particular concern relative to validation and certification of critical systems is caused by electromagnetic disturbances. Sources of electromagnetic disturbances include lightning, High Intensity Radiated Fields (HIRF) caused by RF transmitters and radars, portable electronics devices carried onto the airplane, and electromagnetic incompatibilities of equipment installed on the aircraft [1].

"Soft faults in digital avionics have traditionally been manually corrected. More recently, architecture design measures for the automatic correction of soft faults have begun to be developed. It is perceived that significant benefits can be gained through soft fault protection measures designed into the basic system mechanization. Architecture designs with soft fault protection provide the ability to tolerate disruption of either inputoutput data or internal computation. Fault clearing and computation recovery must be rapid enough to be "transparent" relative to functional operation and flight deck effect." [2]

\section{RCS}

"An example of an architectural soft fault protection philosophy in the design of computing platforms is the Aircraft Information Management System (AIMS) used on Boeing 777 aircraft. All computing and V/O management resources are lock-step compared on a processor cycle-by-cycle basis. In this approach, if a soft or hard fault event occurs, the processor is interrupted and service handlers take control and no data can be exported." [2]

The first stage of a prototype computing platform for fast recovery from soft faults has been developed for NASA. This prototype includes patented technology for transparent soft fault recovery that had not been built or tested before. These concepts were implemented and integrated into the basic lock-step processing module of the AIMS architecture. This prototype was delivered to NASA for evaluation at the Langley Research Center (LaRC) system integration facility. A general illustration of a computing platform with transparent recovery elements is depicted in Figure 1.

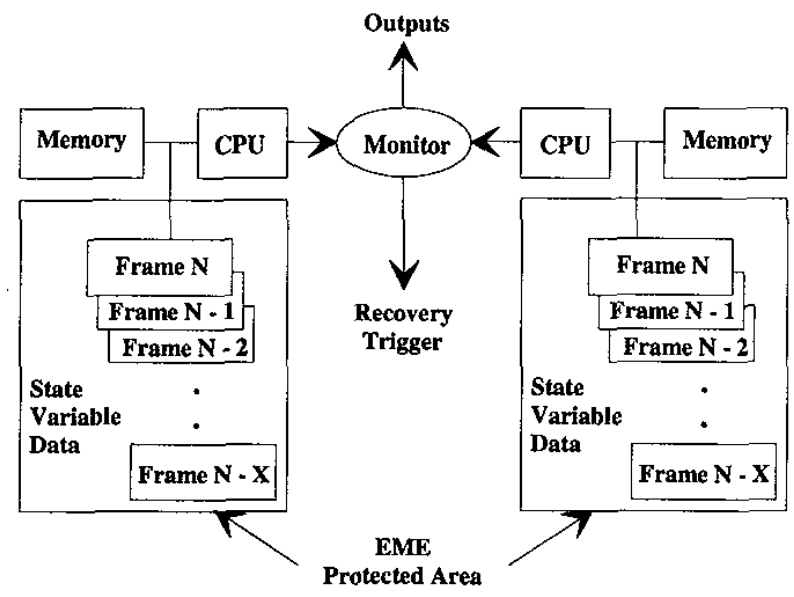

Figure 1. Rapid Recovery Upset Detection and Recovery Flow

Such a platform is intended to provide soft fault recovery that would be virtually software independent and 
transparent to a system function. As shown in Figure 1, the rapid recovery concept involves protecting and recovering the significant state variables of a system function. An aircraft autoland (arm, capture and track) function is being used for evaluation of the rapid recovery concept. Since this is the only function being implemented in the application program software, the partitioning portion of the platform SAFEbus technology was not employed. Communication with the LaRC host system integration facility is intended to be accomplished primarily through an optical 429 bus structure [2].

\section{System Architecture}

The Closed-Loop architecture consists of the RCS, a VME-based optic card for conversion of data between electrical and optical signals, a flight simulation host processor, and a development environment. Since available PCs are very fast, powerful and relatively inexpensive, it seemed practical to attempt to perform the flight simulation operation, as well as collection and display of data on one PC, Figure 2. The challenge, therefore, was proving the feasibility of managing such system and real-time display of data on a PC.

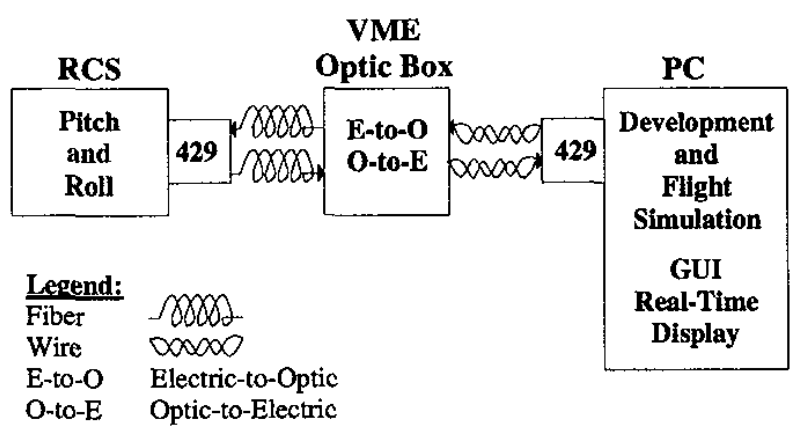

Figure 2. PC-Based Systems.

\section{Software Development}

A real-time Graphical User Interface (GUI) package was developed for the Windows environment using $\mathrm{C} / \mathrm{C}++$ languages [3]. This modularized and userfriendly software consists of 429-interface and flight simulation code. It manages communication between the RCS and the flight simulation code and displays the data in real-time while meeting timing requirements of the RCS, Figure 3. In Figure 3, channel 2 shows the timing diagram of the RCS output and channel 3 is the timing diagram of the PC output. As is evident from this figure, after transmission of the data at the required time, the $\mathrm{PC}$ has ample time to store and display data before the next interval.

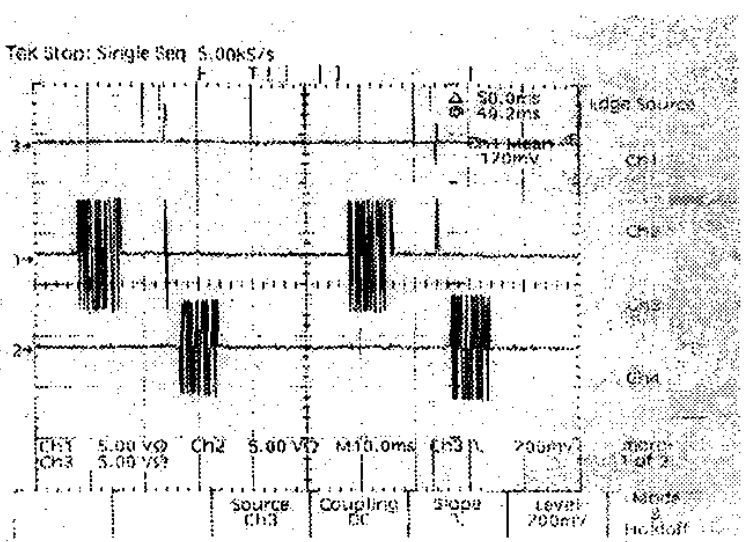

\section{Figure 3. RCS Communication Timing Diagram.}

This GUI software consists of many windows that depict Closed-Loop System activities in real-time. Roll and Aileron command (DELAC), Pitch and Elevator command (DELEC), and Altitude (ALT) and Radio Altitude (HRALT) are displayed in three different windows to provide the user with visual correlation between the RCS inputs and their corresponding output commands. The lateral position (YCG), throttle (THROT), and yaw (YAW) are also displayed separately. In addition to an error-monitoring window, there are four other widows that display the discrete values reported by RCS. As a result, this software monitors all activities of the Closed-Loop System. This real-time monitoring capability is essential for testing the system in the presence of RF, Figure 4.

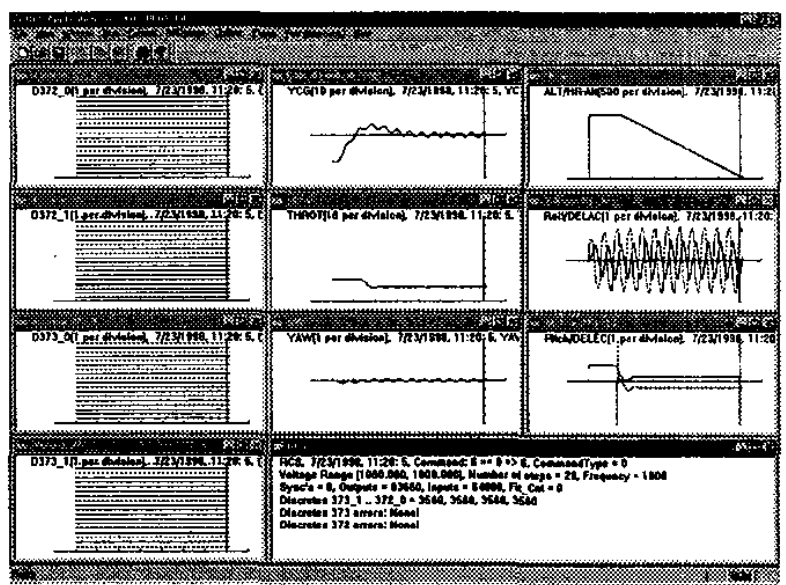

Figure 4. RCS-Win Software. 
Through this user-friendly software, test conditions are specified and data are collected and recorded for future reference. Also, for software fault injection, the exact timing of the fault can be specified.

\subsection{Data Management}

The data along with the test conditions such as the RCS control flags, field strength range, and initial frequency are stored on the hard disk. Each file is approximately 2.4 Mbyte. Removable hard drives and a CD-ROM writer are used for archival storage. The collected data are in ARINC format [4] in order to minimize the storage requirements. The user interface software is then used to convert these data files to the desired format for post-test analysis purposes.

\section{Tests}

Tests for the RCS are being conducted in two phases. Phase one consists of testing under ideal conditions where HIRF is absent. Phase two consists of testing in the HIRF chambers and in the presence of RF. The specific goals are to characterize the RCS functionality and the RCS upset recovery scheme, to verify control laws and flight simulation integrity, and to assess RCS performance under various conditions.

Phase one was necessary in order to resolve discrepancies in the Interface Control Document (ICD) and to debug the system as a whole. Completion of phase one also led to the detection of errors in the implementation of the flight control codes in the RCS [3].

Preliminary results of phase two of the tests are presented in the following sections of this paper.

\subsection{Test Environment}

The reverberation chambers of the HIRF facility at NASA LaRC provide the test environment. Within the test chamber are both transmitting and receiving antennas as well as any sensors that might be needed for a specific test. The components of the signal generation and measurement instrumentation consist of a synthesized sweeper for signal generation, a network analyzer, a spectrum analyzer, an oscilloscope, and a high-power amplifier [5, 6]. These devices are software controlled from within the HIRF Laboratory. The advantage of performing tests in modestirred chambers is that the equipment is subjected to fields at all angles of incidence simultaneously. The reverberation chambers within the HIRF Laboratory provide near-homogeneous randomized electromagnetic fields and make it possible to place the entire target unit in the test environment.

During the test, the RCS is placed inside one of the mode-stirred chambers of the HIRF Laboratory and is interfaced to the flight simulation computer. Electrical isolation is achieved via fiber optic cables, Figure 5.

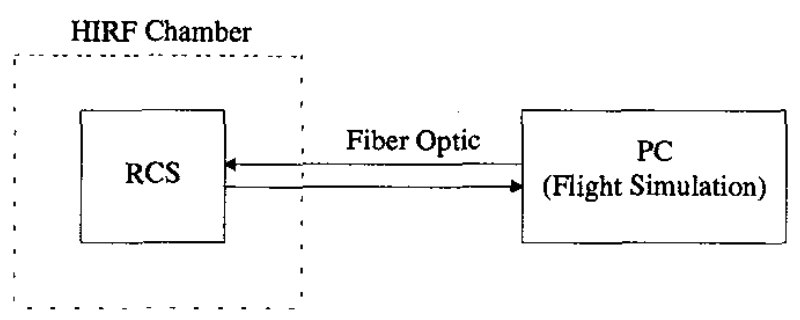

Figure 5. RCS Test Environment.

While testing the RCS in the HIRF chamber, RF and/or field strength are gradually increased until a region of susceptibility is detected. In order to prevent damage to the RCS, RF is turned off for the remainder of the flight as soon as upset is detected. The test is then resumed with finer increments of frequencies and/or field strength in the vicinity of the susceptibility region.

\subsection{Test Approach}

Since the RCS is an expensive one-of-a-kind prototype the test approach was cautiously planned. The RCS was first tested with its shields on for a wide range of frequencies, namely $200 \mathrm{MHz}$ to $1 \mathrm{GHz}$ and $1 \mathrm{GHz}$ to 2 $\mathrm{GHz}$, and field strengths, $100 \mathrm{v} / \mathrm{m}$ to $800 \mathrm{v} / \mathrm{m}$. The RCS proved rather resilient. Although a few regions of susceptibility were detected, the results were not repeatable.

The RCS was then tested with the shields off for the frequency range of $100 \mathrm{MHz}$ to $1 \mathrm{GHz}$ and field strengths of $50 \mathrm{v} / \mathrm{m}$ to $250 \mathrm{v} / \mathrm{m}$.

\subsection{Frequency Coverage}

In order to find all areas of susceptibility, a thorough coverage of all frequencies and field strengths is needed. Such comprehensive testing requires a great deal of time and resources. Since our goal is to characterize the RCS mechanisms, there is no need for such comprehensive coverage nor is there a need for a DO-160 type test. All that is needed are a few areas of susceptibility. A heuristic approach was therefore adopted so that by decreasing the granularity of the frequency coverage, test time and cost could be drastically reduced.

\subsection{Emission Test}

Exploration of the radiated frequencies of the RCS revealed that there are three dominant frequencies emanating from the RCS. These frequencies are due to the internal clocks and oscillators for the optical I/O, RCS processors, and 429 card, at $60 \mathrm{MHz}, 24 \mathrm{MHz}$, and 16 $\mathrm{MHz}$, respectively. The result of the emission test in a 
Semi-Anechoic chamber is shown in Figure 6. This figure corresponds to the emissions of the RCS with its shields removed. The reference line is from section 21 of RTCA/DO-160D environmental conditions and test procedures for airborne equipment.

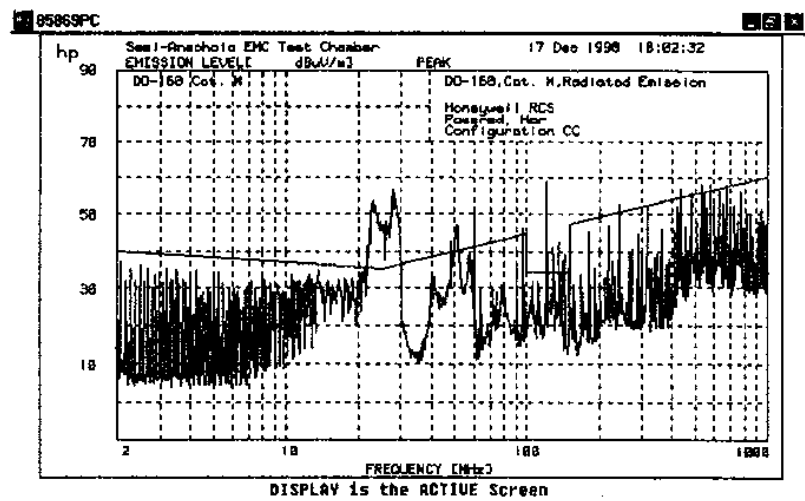

\section{Figure 6. RCS Emission Test Result (Shields off).}

\subsection{Closed-Loop HIRF Data}

Using the data from the Sermi-Anechoic chamber, the test plans were formed to encompass all frequencies within the limitations of the reverberation HIRF facility. During the test, the RCS was continuously exposed to RF. However, for any given frequency, the field strength was gradually stepped up, in 10-second intervals, until the maximum field strength was reached. The 10-second interval was chosen based on the rotation time of the fieldstirrer and to allow time for visual susceptibility monitoring. For every test plan, the range of field strength for all frequencies was the same, therefore, the number of steps from minimum to maximum field strength was also the same in order to maintain consistency for all tests. Table 1 is an example of the first test conditions for continuous HIRF exposure while RCS shields were off.

\section{Table 1. Test Conditions with Continuous HIRF Exposure.}

\begin{tabular}{|c|c|c|c|}
\hline $\begin{array}{c}\text { Frequency } \\
\text { Range } \\
(\mathbf{M H z})\end{array}$ & $\begin{array}{c}\text { Min } \\
\text { Field } \\
(\mathbf{V} / \mathbf{m})\end{array}$ & $\begin{array}{c}\text { Max } \\
\text { Field } \\
(\mathbf{V} / \mathbf{m})\end{array}$ & Recovery \\
\hline F & 50 & 250 & On \\
\hline
\end{tabular}

$$
\begin{aligned}
& \mathrm{F}=\{120 \mathrm{MHz}+60 \mathrm{MHz} * \mathrm{I}, \\
& 120 \mathrm{MHz}+24 \mathrm{MHz} * \mathrm{I}, \\
&112 \mathrm{MHz}+16 \mathrm{MHz} * \mathrm{I}\}<1 \mathrm{GHz} \\
& \mathrm{I}=0,1,2, \ldots
\end{aligned}
$$

Besides unmodulated Continuous Wave (CW $)$, in RCS has been exposed to Square Wave (SQW) modulated radiation. Preliminary data reveal a number of upset regions. Figure 7 shows a crashed flight. Further analyses of the data indicate that the crash was primarily due to upsets on the optical transmitters and receivers. The optical transmitters and receivers were shielded, and the tests were repeated. As a result the upset regions were eliminated. In Figure 7, Roll and DELAC as well as Pitch and DELEC are displayed together to provide visual correlation.

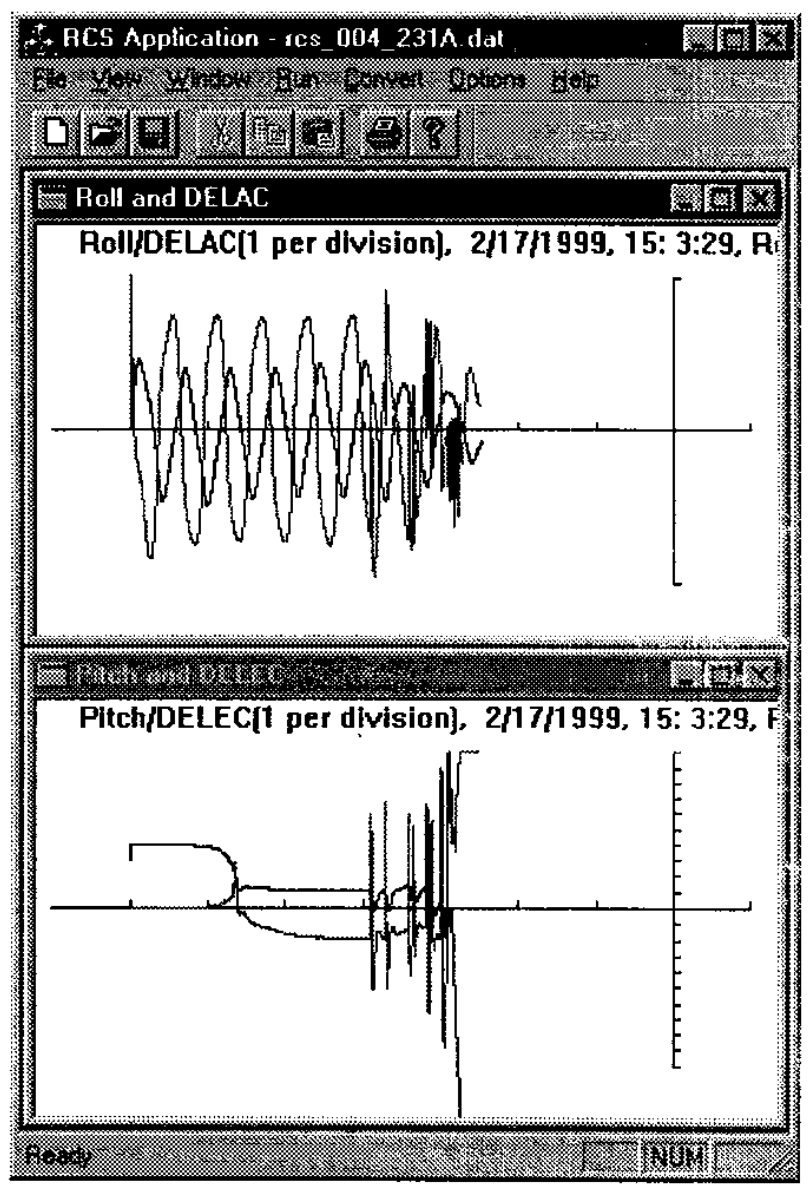

\section{Figure 7. Aileron and Elevator Commands, Communication Failure.}

Since the RCS recovery mechanism was ot triggered during this test, the test range was expanded to cover a wider range of field strengths. However, to keep the number of tests and the required time the same as the previous test, the granularity of the field strengths was decreased. Table 2 is an example of the second test conditions for continuous HIRF exposure while RCS shields were off.

Analyses of the data revealed a number of upset regions, Figure 8 . Further analyses indicate that the upsets 
were internal to the RCS and the recovery mechanism was indeed initiated.

\section{Table 2. Test Conditions with Continuous HIRF Exposure.}

\begin{tabular}{|c|c|c|c|}
\hline $\begin{array}{c}\text { Frequency } \\
\text { Range } \\
(\mathbf{M H z})\end{array}$ & $\begin{array}{c}\text { Min } \\
\text { Field } \\
(\mathbf{V} / \mathbf{m})\end{array}$ & $\begin{array}{c}\text { Max } \\
\text { Field } \\
(\mathbf{V} / \mathbf{m})\end{array}$ & Recovery \\
\hline $\mathrm{F}$ & 100 & 1000 & On \\
\hline
\end{tabular}

$$
\begin{aligned}
\mathrm{F}=\{120 \mathrm{MHz}+60 \mathrm{MHz} * \mathrm{I} \\
120 \mathrm{MHz}+24 \mathrm{MHz} * \mathrm{I} \\
112 \mathrm{MHz}+16 \mathrm{MHz} * \mathrm{I}\}<1 \mathrm{GHz} \\
\mathrm{I}=0,1,2, \ldots
\end{aligned}
$$

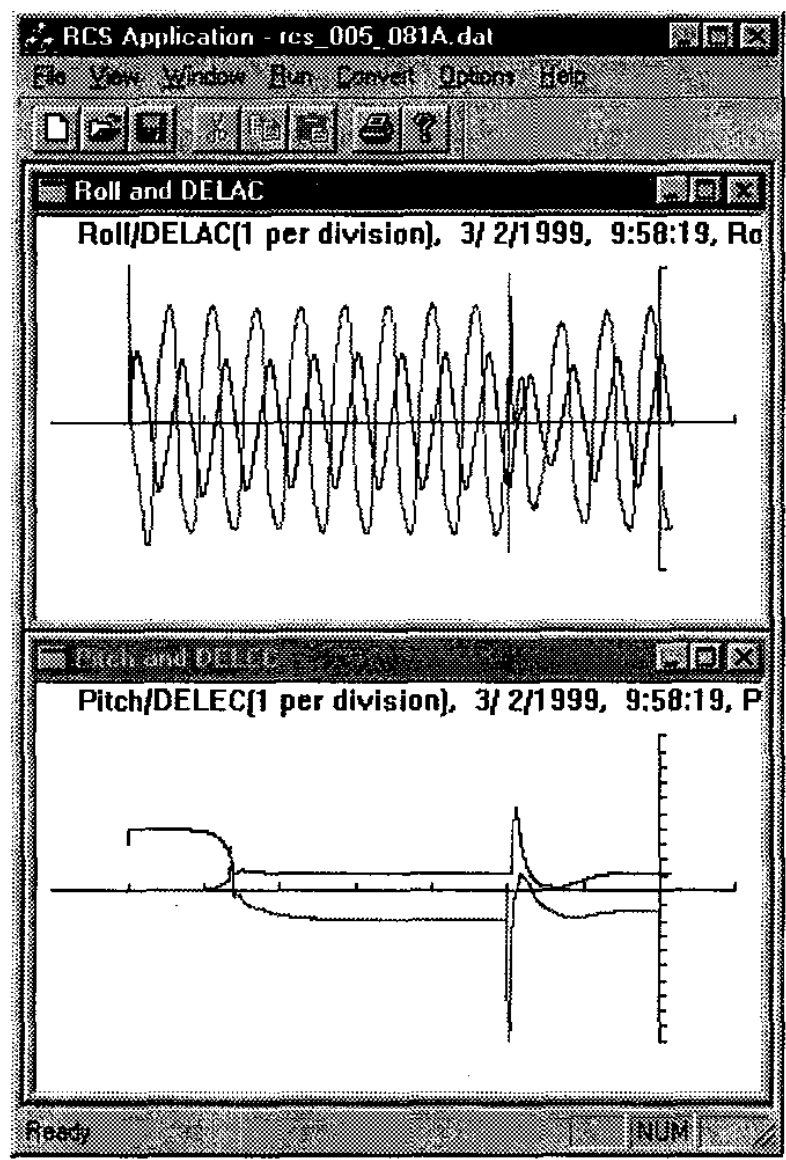

Figure 8. Aileron and Elevator Commands (DELAC and DELEC), HIRF-Induced Fault.

Although the RCS recognized the faults and its recovery mechanism was triggered, it is difficult to determine the exact cause or component responsible for the failure because of the limited accessibility inside the RCS.

\section{Software Fault Injection}

In order to characterize the RCS recovery scheme, a series of tests were conducted in which faults were introduced to the RCS by setting the appropriate flags that would trigger the RCS recovery mechanism. Using the GUI-based software and in the absence of HIRF, faults were injected into the RCS at specific times. These tests were concentrated on four regions of flight: before glide slope engagement, at and in the vicinity of glide slope engagement, after glide slope engagement, and approaching the runway.

\section{RCS Performance Measures}

Analyses of the collected data from the software fault injection tests reveal that the RCS recovery mechanism takes about $360 \mathrm{~ms}$ to complete, while on the average it takes about 30 seconds for the airplane flight attitude to return to normal. The analyses further reveal that the RCS and airplane attitude recovery times are independent of the flight mode. A closer look at the response of the Aileron Command (DELAC) for the case of a software injected fault from prior to fault injection and until after the RCS internal recovery process is depicted in Figure 9. As shown in Figure 9, the flight continued (solid line) for the duration of the RCS recovery using the old values. Upon recovery, however, abrupt command output transitions upset the flight attitude, which in turn takes approximately 30 second to correct. These performance measures correlate to the measured values for the case of HIRF-induced faults, Figure 8.

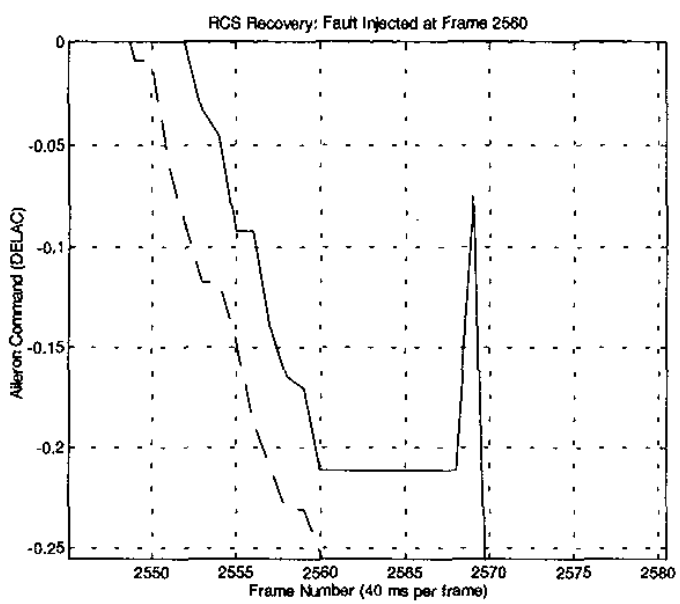

Figure 9. RCS Recovery Triggered. (Dashed line is the reference).

Another set of tests was conducted to further observe the effects of communication errors on the RCS 
recovery scheme. For these tests, communication lines to the RCS were disconnected and reconnected for various time delays. Regardless of the duration of the communication breakdown and upon reestablishing communication, the flight continued with minor interruptions in the control laws and airplane attitude. Figure 10 is an example of a communication breakdown of one-second duration. Since the RCS recovery mechanism was not triggered, this observed response is most likely due to the natural behavior of the control laws and the airplane simulation, and probably had little to do with the special internal mechanisms of the RCS.

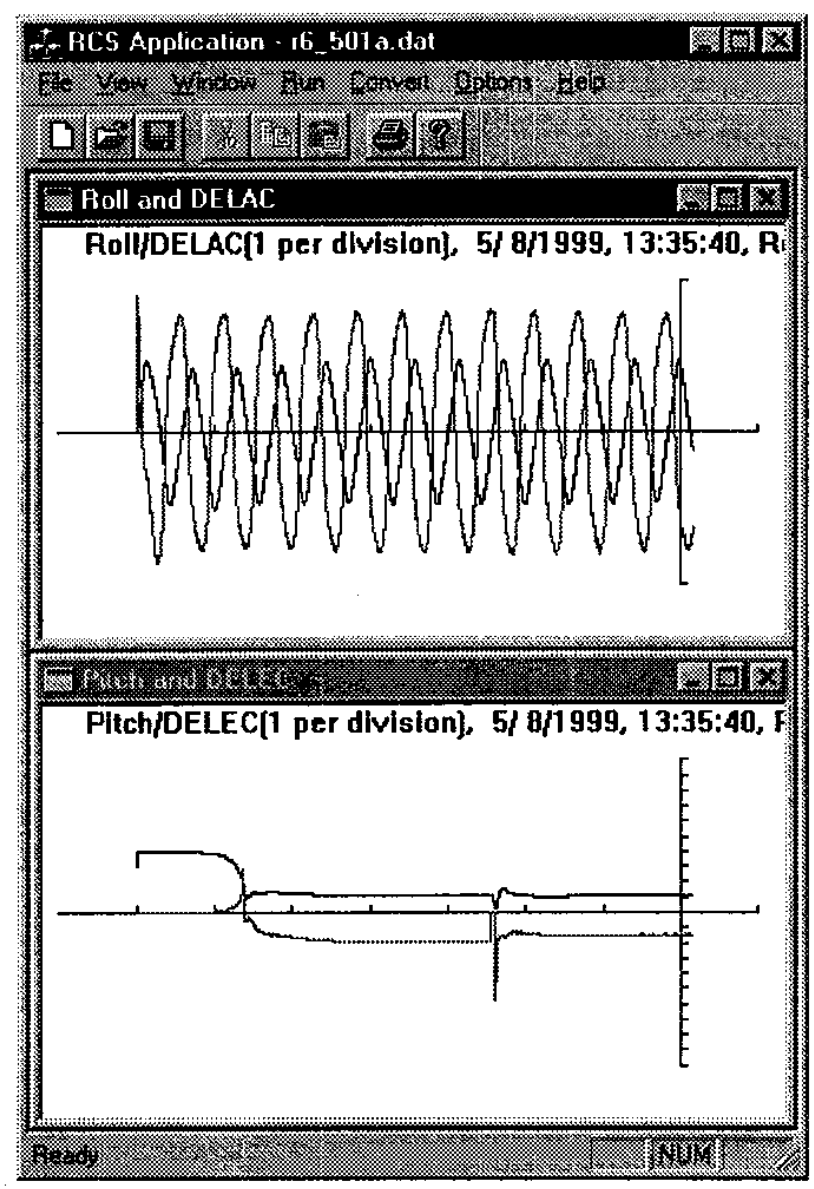

Figure 10. Communication Interruption of One Second.

Comparison of the behavior of the system during a HIRF induced fault to that of a communication breakdown, Figures 8 and 10, respectively, reveals that internal faults have noticeable effects on the performance of the RCS. Furthermore, the fault injection experiments seem to confirm this finding. Questions arise as to whether this is due to implementation of the control laws in the RCS, the backup mechanism of the airplane states, the recovery procedure, or the hardware limitation of this prototype. The search for answers to these questions will help direct future plans for this work.

\section{Summary}

The design and development of a Closed-Loop System to study and evaluate the performance of the advanced RCS architecture when subjected to EME is presented. The integration of this hardware proved to be a major undertaking. During this process a number of bugs in the 737-control law implementation of the RCS were found that are being resolved. In support of this ClosedLoop System, a Windows-based software package was developed to handle the time critical communication of data and commands between the RCS and flight simulation code, in real-time while meeting the stringent hard deadlines. This package consists of an ARINC 429 bus driver, flight simulation code and GUI-based displays of the key elements of the control laws, as well as the airplane attitude. As a result, this package enables the researchers to monitor all activities of the airplane during the flight in real time. The real-time capability of this package is crucial during the EME testing of the hardware. The upset recovery scheme of the RCS is characterized and performance of the RCS under various conditions is discussed.

\section{References}

[1] Celeste M. Belcastro, "Closed-Loop HIRF Experiments Performed on a Fault Tolerant Flight Control Computer," 16th AIAA/IEEE DASC, Irvine, CA, October 1997.

[2] R.F. Hess, "Computing Platform Architectures for Robust Operation in the Presence of Lightning and Other Electromagnetic Threats," 16th AIAA/IEEE DASC, Irvine, CA, October 1997.

[3] Mahyar Malekpour, "Evaluation of Honeywell Recoverable Computer System (RCS) in Presence of Electromagnetic Effects," $17^{\text {th }}$ AIAA/IEEE DASC, Seatile, CA, October 1998.

[4] ARINC Specification 429P1-15, September 1, 1995.

[5] Daniel M. Koppen, “Open-Loop HIRF Experiments Performed on a Fault Tolerant Flight Control Computer," 16th AIAA/IEEE DASC, Irvine, CA, October 1997.

[6] Rueben A. Williams, "The NASA High Integrity Radiated Fields Laboratory," 16th AIAA/IEEE DASC, Irvine, CA, October 1997. 